Full length article

\title{
Laparoscopic lateral suspension with mesh for apical and anterior pelvic organ prolapse: A prospective double center study
}

\section{Liliana Mereu $^{\mathrm{a}, *}$, Saverio Tateo $^{\mathrm{a}}$, Maurizio Nicola D’Alterio ${ }^{\mathrm{a}, \mathrm{e}}$, Eleonora Russo ${ }^{\mathrm{b}}$, Andrea Giannini ${ }^{\mathrm{b}}$, Paolo Mannella ${ }^{\mathrm{b}}$, Riccardo Pertile ${ }^{\mathrm{c}}$, Tommaso Cai ${ }^{\mathrm{d}}$, Tommaso Simoncini ${ }^{\text {b }}$}

a Department of Obstetrics and Gynecology, S Chiara Hospital, Trento, Italy

${ }^{\mathrm{b}}$ Department of Obstetrics and Gynecology, University of Pisa, Italy

${ }^{\mathrm{c}}$ Clinical and Evaluative Epidemiology Department - Trento Health Service, Trento, Italy

d Department of Urology, S Chiara Hospital, Trento, Italy

e University of Cagliari, Italy

\section{A R T I C L E I N F O}

\section{Article history:}

Received 26 May 2019

Received in revised form 8 October 2019

Accepted 17 October 2019

Available online $\mathrm{xxx}$

\section{Keywords:}

Apical defect

Complications

Laparoscopic lateral suspension

Mesh

Pelvic organ prolapse

Recurrences

\begin{abstract}
A B S T R A C T
Objective: The present study analyzed long-term outcomes and complications of laparoscopic lateral suspension (LLS) with mesh to treat apical and anterior pelvic organ prolapse (POP).

Study design: A prospective cohort study on 125 patients with vaginal bulge and apical $+/-$ anterior prolapse scheduled for LLS who consecutively underwent LLS between April 2013 and January 2017 in Gynecologic Department of Santa Chiara Hospital in Trento and University of Pisa.

The main outcome measure was anatomic and symptomatic POP outcome; the secondary outcomes measures were recurrence, reoperation rate, de novo posterior POP and complications. Percentage distribution of the pre- and postoperative POP-Q stages was compared at mean follow-up. Wilcoxon signed rank sum test was used to compare preoperative POP-Q stage and postoperative POP-Q stage ate mean follow up, for each patient (paired data) and for each type of prolapse.

Results: 120 patients were included in the study. At 2 years $89 \%$ of patients were asymptomatic and anatomic success rate was $94.2 \%$ for the anterior compartment, $94.9 \%$ for the apical compartment. Concerning posterior compartment prolapse $2(1.7 \%)$ patients referred stage 3 de novo prolapse during follow-up. The complication rate of Clavien-Dindo $>3$ was $0,8 \%$. Repeat surgery for POP occurred in $6.4 \%$ of cases. The appearance of POP-Q recurrences was concentrated at 6 months follow-up. BMI $>25$ was correlated with de novo posterior compartment appearance during follow-up.

Conclusions: LLS for the treatment of apical and anterior POP is a technique with optimal results in term of safety and effectiveness after 2 years follow-up.
\end{abstract}

(c) 2019 Elsevier B.V. All rights reserved.

\section{Introduction}

POP is a frequent condition and can affect a woman's quality of life. In post-menopausal women, the prevalence of POP is 3-6\% when defined and graded on symptoms compared with $41-50 \%$ when based on examination [1,2]. The anterior compartment is the most frequently reported site of prolapse, it is identified twice more than posterior and three times more than apical compartment defect [3]. Only 10-20\% of affected women, though, seek evaluation for their condition [2]. Although surgery is generally

\footnotetext{
* Corresponding author at: Department of Obstetrics and Gynecology, Ospedale Santa Chiara, Trento Largo Medaglie d'Oro 9, 38122, Trento, Italy.

E-mail address: liliana.mereu@apss.tn.it (L. Mereu).
}

reserved to patients with prolapse symptoms, with advance stage or after failure of conservative treatments, woman's lifetime risk of surgery for POP is $12-19 \%$ [4].

There are various surgical techniques for the correction of POP: fascial vs mesh repair, vaginal vs abdominal route and different abdominal approaches: laparotomy, laparoscopy, robot-assisted laparoscopy. After the FDA warning of 2011 and 2019 concerning POP repair with vaginal mesh, trans-abdominal mesh procedures have reached the more favourable risk-benefit proportion for correction of apical defect. By some, sacrocolpopexy (SCP) is considered the gold standard [2] even if it can be associated with long operative time and learning curve, serious morbidity as vascular and ureteric injuries, lesions of the superior hypogastric plexus, right hypogastric nerve leading to de novo constipation and even if rare spondylodiscitis and lumbar pain [5-7]. 
An alternative procedure to SCP that avoids dissection at the promontory could be LLS. This procedure provides symmetrical lateral tension-free suspension of the central mesh part attached to the vagina and it can be performed with or without uterus preservation or after hysterectomy. Kapandgji described lateral suspension the first time in 1967 [8]. Then Dubuisson modified the technique for laparoscopic approach in 1998 [9], to treat anterior and apical compartment POP, reporting good results in term of absence of complications and POP recurrences after one year of clinical follow-up [10]. Recently Mini-laparoscopic and Robotic approaches have also been described with optimal results in term of feasibility, learning curve and complications [11,12].

The aim of this study was to analyze POP outcomes and safety of LLS for apical and anterior POP.

\section{Material and methods}

This is a prospective cohort study of consecutive patients who underwent LLS for apical and anterior POP between April 2013 and January 2017 at the Gynecological Unit of the S. Chiara Hospital of Trento and at the Division of Obstetrics and Gynecology of the University of Pisa. Inclusion criteria were: symptomatic stage 2 or greater (point $C \geq-1$ pelvic organ prolapse quantification $P O P-Q$ ) apical prolapse (uterovaginal or vault prolapse) with or without anterior compartment prolapse (point $\mathrm{Ba} \geq-1$ pelvic organ prolapse quantification $\mathrm{POP}-\mathrm{Q}$ ) and without symptomatic or significant posterior compartment prolapse (point $\mathrm{Bp}>1$ pelvic organ prolapse quantification POP-Q).

Exclusion criteria: patients who underwent LLS with abdominal fascia fixation of the mesh. Patients with apical $+/$-anterior and posterior prolapse underwent sacrocolpopexy. Informed consent to LLS was obtained from all patients in accordance with local legislation.

Institutional Review Board approval was obtained from both the Ethical Committee of Azienda Provinciale Servizi Sanitari of Trento and the University of Pisa.

Pre-treatment evaluation included: medical history collection, pelvic ultrasound, physical examination, clinical evaluation of pelvic organ support assessed by the Pelvic Organ Prolapse Quantification Grading system (POP-Q). Clinical patient characteristics including age, BMI, menopausal hormonal status, sexual activity, parity, dyspareunia, bladder dysfunctions, intestinal symptoms such as constipation and fecal incontinence, comorbidity and prior surgery for POP were recorded. Intraoperative parameters including concomitant surgery, overall operating time, blood loss, conversion rate, post-operative pain, complications, time to discharge and recurrence were recorded as well. The estimated blood loss (EBL) was calculated by the difference in the total amounts of suctioned and irrigation fluids. Operative time was defined as the time from skin incision to the skin closure. Perioperative, early postoperative complications ( $\leq 30$ days) and late postoperative complications ( $>30$ days) were recorded. Dyspareunia was recorded using a Numerical Rating Scale (NRS) from 1 to 10 (dyspareunia $>4$ has been considered clinically relevant). Bladder dysfunctions were classified in urinary stress incontinence, urge incontinence and voiding dysfunction. Postvoid residual volume meausrement and urodynamic evaluation have been performed when required in relation to patients' symptoms [13]. The Incontinence Impact Questionaire 7 (IIQ7) was used to assess the impact of UI [14], defecatory functional status was assessed by the Wexner constipation score [15]. Intraoperative complications were scored according to Classification of Intraoperative Complications [16] and postoperative complications according to the "Clavien-Dindo classification of surgical complications scale" [17]. Mesh-related complications were classified using the IUGA/ICS complication classification [18]. Postoperative pain assessment was performed using NRS. Systematic postoperative clinical and symptomatic evaluation were performed at 6 weeks, 6 months, one and 2 years. Anatomic cure was define as POP-Q Ba, C, Bp < $+1 \mathrm{~cm}$, while subjective outcomes were determined by asking to the patients about presence of bulge symptoms. The main outcomes measures were anatomic and symptom outcomes; the secondary outcome measures were recurrence, reoperation rate, de novo posterior POP and complications.

In absence of uterine disease, hysteropexy was the first choice. LLS technique described by Dubuisson et al. [19] has been adopted not only for the laparoscopic but also for the mini-laparoscopic and robotic approach $[16,20]$. In all cases a titanium-coated macroporous polypropylene mesh (TiLOOP ${ }^{\mathbb{R}}$ Dubuisson) was used.

\section{Statistical analysis}

An initial overview of the sample was obtained through the creation of a frequency distribution table (absolute and percentage) of demographic and clinical variables. The mean standard deviation and median values were presented for the age of patients.

The wilcoxon signed rank sum test was used to compare preoperative POP-Q stage and postoperative POP-Q stage at mean follow up, for each patient (paired data) and for each type of prolapse. This statistical test has been chosen because the difference between the two variables is not normally distributed and the variables are ordinal. Mean values and SD are also presented for each group.

The significance of the association between the postoperative POP-Q stage and couples of times of follow-up ( 6 weeks vs. 6 months and 6 months vs. 12 months) was analyzed with Fisher-s Exact test, considering the association significant when the p-value was $<0.05$.

The univariate analysis took into account the associations between independent variables (such as type of minimal invasive approach, BMI classes, age classes, parity, hormonal status and prior POP surgery) and the outcome variables (type of recurrence of POP, repeat surgery for POP, surgery for different site, surgery for complications, vaginal bulge, de novo symptomatic posterior and asymptomatic $\mathrm{POP} \geq 3$ ): these associations were all invariably studied with Fisher's Exact test.

\section{Results}

125 patients consecutively underwent to LLS; 5 patients were excluded because submitted to a modified LLS with mesh attached to abdominal fascia.

Patient characteristics and preoperative symptoms are shown in Table 1.

Indication to LLS was, in all cases, the presence of vaginal bulge and apical $+/$ - anterior prolapse. Apical prolapse $\geq 3$ was present preoperatively in $96(80,6 \%)$ patients, anterior compartment defect $\geq 3$ in $86(72.3 \%)$ patients and vaginal bulge in all cases.

Intraoperative findings are shown in Table 2.

The Foley catheter was removed on the morning after surgery. Mean hospital stay was 2.1 days (range 1-10). Mean length of postoperative follow up was 20 months: all patients had a followup at 6 weeks, 6 and 12 months, and 89 at 24 months.

Postoperative complications at 1 month occurred in 7 cases, 2 patients grade 1: pain and vaginitis, 1 patient grade 2 : pyelonephritis requiring IV antibiotics therapy and 10 days of hospitalization, 1 patients grade 3: 1 mesh erosion requiring vaginal excision of mesh exposure. No grade IV complications occurred.

Concerning urogynecological de novo symptoms: 7 (5.8\%) urgency, $3(2.5 \%)$ stress urinary incontinence (SUI), 3 (2.5\%) 
Table 1

Patients' characteristics.

\begin{tabular}{ll}
\hline & $\begin{array}{l}\text { All patients } \\
(n=120)\end{array}$ \\
\hline Caucasian ethnicity, n (\%) & $120(100)$ \\
Age (years), mean \pm SD (median) & $60 \pm 9.85(61)$ \\
BMI $\left(\mathrm{kg} / \mathrm{m}^{2}\right)<25, n(\%)$ & $66(55)$ \\
BMI $\left(\mathrm{kg} / \mathrm{m}^{2}\right)>25, n(\%)$ & $59(49.1)$ \\
& \\
Parity, $n(\%)$ & \\
Nulliparous & $1(0.8)$ \\
Multiparous & $119(99.21$ \\
& \\
Number of vaginal deliveries, $n(\%)$ & \\
One & $42(35)$ \\
Two or more & $81(67.2)$ \\
Menopausal, n (\%) & $100(83.3)$ \\
HRT, n (\%) & $7(5.8)$ \\
Sexual activity, n (\%) & $96(80)$ \\
Dyspareunia $>4, \mathrm{n}(\%)$ & $11(9.1)$ \\
Voiding obstruction, n (\%) & $4(3.3)$ \\
Stress Incontinence, n (\%) & $0(0)$ \\
Urgency incontinence, n (\%) & $11(9.1)$ \\
Constipation, n (\%) & $0(0)$ \\
Fecal incontinence, n (\%) & $0(0)$ \\
Prior POP surgery, n (\%) & $13(10.8)$ \\
Prior urinary incontinence surgery, n (\%) & $3(2.5)$ \\
Prior hysterectomy, n (\%) & $7(5.8)$ \\
\hline
\end{tabular}

$\mathrm{BMI}=$ Body Mass Index.

HRT = Hormone Replacement Therapy.

Voiding obstruction includes symptoms of hesitancy, weak stream, incomplete empyting.

Table 2

Surgical findings.

\begin{tabular}{ll}
\hline & All patients $(n=120)$ \\
\hline Approaches & \\
Standard laparoscopy, n(\%) & $40(33.3)$ \\
Mini-laparoscopy, n (\%) & $20(16.6)$ \\
Robotic assisted laparoscopy, n(\%) & $60(50.0)$ \\
& \\
Suspension & \\
Hystero & $105(87.5)$ \\
cervical & $8(6.6)$ \\
Vaginal vault & $7(5.8)$ \\
Anterior mesh, n (\%) & $120(100 \%)$ \\
Posterior mesh n, (\%) & $0(0 \%)$ \\
Concomitant procedures, n (\%) & $45(37.5)$ \\
Total hysterectomy, n & 1 \\
Subtotal hysterectomy, n & 7 \\
Myomectomy, n & 2 \\
Salpingoophorectomy, n & 12 \\
Salpingectomy, $\mathrm{n}$ & 7 \\
Cervical amputation & 7 \\
Stapled transanal rectal resection, n & 2 \\
Hysteroscopic polipectomy, n & 2 \\
Adhesiolysis, n & 5 \\
Mean operative time, mean (range) & $120(63-280)$ \\
Intraoperative complications, n (\%) & $0(0)$ \\
Conversion to laparotomy n, (\%) & $0(0)$ \\
\hline
\end{tabular}

transient voiding obstruction cases occurred in previously asymptomatic patients. No patient developed de novo constipation, fecal incontinence or dyspareunia and 11 women who had preoperative dyspareunia improved after surgery. Urgency incontinence was resolved with medical treatment (topic estrogen) and the three cases of SUI did not require any surgery at the moment.

At 2 years $89 \%$ of patients were asymptomatic and anatomic success rate was $94.2 \%$.

Anatomical results are detailed in Table 3.

During the follow up we observed the following recurrences: 6 (5\%) stage 3 anterior compartment, 1 (0.8\%) stage 4 anterior compartment, 5(4.2\%) stage 3 apical compartment, 1 (0.8\%) stage 4 apical compartment. Concerning posterior compartment prolapse we had 2 (1.6\%) stage 3 and no stage 4 de novo prolapses. Vaginal bulging occurred in $14(11.7 \%)$ cases and de novo symptomatic posterior prolapse in $1(0.8 \%)$ women. A total of 8 (6.7 \%) patients underwent subsequent SCP because of symptomatic prolapse: 1 patient for concomitant anterior, apical and posterior prolapse stage 4, 6 for anterior and apical stage 3 and 1 for posterior stage 3 . No patient underwent subsequent surgery for stress incontinence.

The recurrences of stage 3 and 4 are concentrated at 6 months follow-up with a statistical significantly difference from the recurrence at 6 weeks and 12 months (anterior compartment $\mathrm{p}=0.007$, apical compartment $\mathrm{p}=0.001$, posterior compartment $\mathrm{p}=0.032$ ). Table 4 . No significantly difference has been found at 12 and 24 months.

Univariate analysis shows that only BMI $>25$ is correlated with de novo posterior compartment appearance during follow up $(p=0.0278)$, while neither patients characteristics nor type of surgery seems to influence anterior or apical prolapse recurrences, vaginal bulge, repeat surgery for POP, or surgery complications $(\mathrm{p}>0.05)$.

\section{Discussion}

The present series of 120 patients mirrors a previous experience of two centres that already published articles on LLS with mesh in the treatment of genital prolapse $[11,12,20]$.

In our opinion, indication is crucial for this kind of correction; indeed, as already suggested by Dubuisson et al, relevant surgical indications are hysterocele and/or cystocele; the procedure is not indicated in case of predominant rectocele, enterocele and/or pelvic floor insufficiency $[10,21]$. For this reason, we excluded patients with apical and posterior defect, to whom we recommend SCP, which provides reinforcement in the rectovaginal space and a posterior direction of the vaginal axis [19]

LLS allows preservation of the uterus, thus providing a significant surgical, functional and psychological benefit in term of safety, reduction of operative time, anatomy preservation and patient satisfaction [10]. In the present study 8 patients underwent supracervical hysterectomy for symptomatic uterine myomas and 1 patient underwent total hysterectomy for absence of preoperative endometrial assessment.

LLS can be performed with different approaches [11,12,21]. We did not find statistical difference in term of outcomes among women underwent standard laparoscopy, minilaparoscopy or robotic.

For all patients a titanium-coated macroporous polypropylene mesh (TiLOOP ${ }^{\mathbb{R}}$ Dubuisson) was used: it is a hydrophilic material that adheres more stably to the vaginal wall, with an easier manipulation, optimal tissue engraftment and enhanced biocompatibility [22-24]. In the present series only one $(0.8 \%)$ mesh erosion occurred, one month after surgery; the incidence is significantly lower compared with the $4 \%$ cumulative incidence of mesh erosion reported in a systematic review after abdominal surgery with mesh during a 2-years follow up [25] and of 4,3\% after 417 laparoscopic lateral suspension procedures [14] where both microporouse and macroporouse mesh was utilized. It is well known that Polypropylene is the mesh material that allows the development of well-organized, fibrous, mature connective tissue [26-28] and a titanium layer over polypropylene seems to improve the benefits of polypropylene, as demonstrated in hernia repair [24], notably reducing the risk of extrusion or exposure.

The overall objective success rate of $94 \%$ and the overall subjective cure rate of $89 \%$ at 19 months of follow-up in both anterior and apical compartment are comparable to the success 
Table 3

Pre- and postoperative POP-Q stage at mean follow up.

\begin{tabular}{|c|c|c|c|}
\hline POP-Q & Preoperative n (\%) & $\begin{array}{l}\text { Postoperative } \\
\text { at an average of } 20 \text { months n (\%) }\end{array}$ & $\begin{array}{l}p \text {-value Wilcoxon signed } \\
\text { rank sum test }\end{array}$ \\
\hline \multicolumn{4}{|l|}{ Point Ba } \\
\hline 0 & $1(0.8)$ & $88(73.3)$ & \\
\hline$<-1 \mathrm{~cm}$ & $7(5.8)$ & $15(12.5)$ & \\
\hline$\geq-1 \mathrm{~cm}$ to $\leq 1 \mathrm{~cm}$ & $20(16.6)$ & $10(8.4)$ & $<0.0001$ \\
\hline$>1 \mathrm{~cm}$ to $<($ TVL- 2$) \mathrm{cm}$ & $69(57.6)$ & $6(5)$ & \\
\hline$\geq(\mathrm{TVL}-2) \mathrm{cm}$ & $23(191)$ & $1(0.8)$ & \\
\hline Mean (SD) & $2.9(0.8)$ & $0.5(0.9)$ & \\
\hline \multicolumn{4}{|l|}{ Point C } \\
\hline 0 & $0(0.0)$ & $109(90.8)$ & \\
\hline$<-1 \mathrm{~cm}$ & $0(0.0)$ & $3(2.5)$ & \\
\hline$\geq-1 \mathrm{~cm}$ to $\leq 1 \mathrm{~cm}$ & $\leq 37(30.8)$ & $2(1.6)$ & $<0.0001$ \\
\hline$>1 \mathrm{~cm}$ to $<($ TVL- 2$) \mathrm{cm}$ & $48(40.0)$ & $5(4.1)$ & \\
\hline$\geq($ TVL-2) $\mathrm{cm}$ & $35(29.1)$ & $1(0.8)$ & \\
\hline Mean (SD) & $3.0(0.8)$ & $0.2(0.7)$ & \\
\hline \multicolumn{4}{|l|}{ Point Bp } \\
\hline 0 & $75(62.5)$ & $72(60.0)$ & \\
\hline$<-1 \mathrm{~cm}$ & $40(33.3)$ & $26(21.6)$ & \\
\hline$\geq-1 \mathrm{~cm}$ to $\leq 1 \mathrm{~cm}$ & $5(4.1)$ & $20(16.6)$ & \\
\hline$>1 \mathrm{~cm}$ to $<($ TVL- 2$) \mathrm{cm}$ & $0(0.0)$ & $2(1.6)$ & 0.02 \\
\hline$\geq($ TVL-2) $\mathrm{cm}$ & $0(0.0)$ & $0(0.0)$ & \\
\hline Mean (SD) & $0.4(0.6)$ & $0.6(0.8)$ & \\
\hline
\end{tabular}

Table 4

De novo appearance of POP during follow-up.

\begin{tabular}{|c|c|c|c|c|}
\hline & \multicolumn{2}{|c|}{$\begin{array}{l}\text { Comparison between Postoperative } 6 \text { weeks and } \\
\text { Postoperative } 6 \text { months }\end{array}$} & \multicolumn{2}{|c|}{$\begin{array}{l}\text { Comparison between Postoperative } 6 \text { months and } \\
\text { Postoperative } 12 \text { months }\end{array}$} \\
\hline & $\begin{array}{l}\text { Postoperative } \\
6 \text { weeks, }(n=120) n(\%)\end{array}$ & $\begin{array}{l}\text { Postoperative } \\
6 \text { months } \\
(\mathrm{n}=120) \mathrm{n}(\%)\end{array}$ & $\begin{array}{l}\text { Postoperative } \\
6 \text { months }(n=120) n(\%)\end{array}$ & $\begin{array}{l}\text { Postoperative } \\
12 \text { months } \\
(\mathrm{n}=120) \mathrm{n}(\%)\end{array}$ \\
\hline \multicolumn{5}{|l|}{ Point Ba } \\
\hline$<-1 \mathrm{~cm}$ & $13(10.8 \%)$ & $4(3.3 \%)$ & $4(3.3 \%)$ & $5(4.1 \%)$ \\
\hline$\geq-1 \mathrm{~cm}$ to $\leq 1 \mathrm{~cm}$ & $10(8.3 \%)$ & $6(5 \%)$ & $6(5 \%)$ & $2(1.6 \%)$ \\
\hline$>1 \mathrm{~cm}$ to $<($ TVL- 2$) \mathrm{cm}$ & $0(0.0 \%)$ & $6(5 \%)$ & $6(5 \%)$ & $0(0.0 \%)$ \\
\hline$\geq(\mathrm{TVL}-2) \mathrm{cm}$ & $0(0.0 \%)$ & $1(0.8 \%)$ & $1(0.8 \%)$ & $0(0.0 \%)$ \\
\hline p-value Fisher's Exact test & 0.007 & & 0.11 & \\
\hline \multicolumn{5}{|l|}{ Point C } \\
\hline$<-1 \mathrm{~cm}$ & $7(5.8 \%)$ & $0(0.0 \%)$ & $0(0.0 \%)$ & $1(0.8 \%)$ \\
\hline$\geq-1 \mathrm{~cm}$ to $\leq 1 \mathrm{~cm}$ & $3(2.5 \%)$ & $2(1.6 \%)$ & $2(1.6 \%)$ & $0(0.0 \%)$ \\
\hline$>1 \mathrm{~cm}$ to $<(\mathrm{TVL}-2) \mathrm{cm}$ & $0(0.0 \%)$ & $5(4.1 \%)$ & $5(4.1 \%)$ & $0(0.0 \%)$ \\
\hline$\geq(\mathrm{TVL}-2) \mathrm{cm}$ & $0(0.0 \%)$ & $1(0.8 \%)$ & $1(0.8 \%)$ & $0(0.0 \%)$ \\
\hline$p$-value Fisher's Exact test & 0.001 & & 0.011 & \\
\hline \multicolumn{5}{|l|}{ Point Bp } \\
\hline$<-1 \mathrm{~cm}$ & $16(13.3 \%)$ & $9(7.5 \%)$ & $9(7.5 \%)$ & $2(1.6 \%)$ \\
\hline$\geq-1 \mathrm{~cm}$ to $\leq 1 \mathrm{~cm}$ & $4(3.3 \%)$ & $12(10 \%)$ & $12(10 \%)$ & $3(2.5 \%)$ \\
\hline$>1 \mathrm{~cm}$ to $<(\mathrm{TVL}-2) \mathrm{cm}$ & $0(0.0 \%)$ & $1(0.8 \%)$ & $1(0.8 \%)$ & $0(0.0 \%)$ \\
\hline$\geq($ TVL-2) $\mathrm{cm}$ & $0(0.0 \%)$ & $0(0.0 \%)$ & $0(0.0 \%)$ & $0(0.0 \%)$ \\
\hline$p$-value Fisher's Exact test & 0.032 & & 0.883 & \\
\hline
\end{tabular}

rate following SCP (97.7\% and $92 \%$ respectively) [6]. Postoperative vaginal bulge was reported by $11 \%$ of patients. It is a better result if compared with $21.6 \%$ described in 417 laparoscopic lateral suspension procedures for anterior, apical and posterior prolapse by Veit-Rubin [29]. Only 8 patients (6.6\%) repeated surgery for POP during two years of follow-up, which is consistent with the findings of several investigators who reported re-operation rates from $3.4-11 \%[29,30]$. These low rates of objective and symptomatic prolapse recurrences indicate excellent outcomes of this technique for the treatment of apical and anterior defect according to the criteria described by Barber et al. [2].

With regard to the posterior compartment, we found de novo posterior prolapse in $3(2.5 \%)$ patients; 1 de novo symptomatic and 2 de novo posterior POP-Q $>3$. Two patients underwent surgery during follow-up for symptomatic posterior prolapse (one with concomitant apical and anterior POP and one with only posterior POP).

We found an association between appearance of postoperative POP and time to follow up, the recurrence of anterior and apical prolapse and the appearance of asymptomatic stage $1 / 2$ posterior prolapse were detected significantly more often during the 6 month-follow up visit.

The univariate analysis reveals that only BMI $>25$ is related to de novo posterior compartment defect $>2$, while the type of surgical approach, age, parity, menopausal hormonal status, prior POP surgery seem not to have a significant association with POP recurrence, reintervention or POP symptoms. These findings suggest that we could consider a concomitant preventative correction of the posterior compartment in selected groups of patients or we could advise weight loss prior to surgical POP correction. 
These results suggest that the suspension axis of a lateral mesh does not lead to enterocele or Pouch of Douglas hernia in patients treated for apical and anterior prolapse; the identification, during the follow up of a $19 \%$ of asymptomatic posterior POP-Q $=2$ would demonstrate that LLS reveals the presence of pre-existing posterior defect rather than determines its appearance. While vaginal sacrospinous or sacrocolpopexy clearly divert the physiologic axis of the vagina, LLS seems not to change vaginal anatomy [31]. We do not know whether or not the "correct" vaginal axis impacts pain or sexual function. As these anatomic considerations are merely hypothesises, further studies are needed to clarify these aspects.

The absence of dyspareunia after this surgical procedure seems to preserve the normal sexual function. During the follow up we also found a low rate (10\%) of urinary symptoms and no de novo intestinal symptoms such as constipation and fecal incontinence.

In our cohort of patients who underwent standard LLS, postoperative Clavien-Dindo grade 3 complications occurred only in $1 / 119(0.8 \%)$ of cases.

The low incidence of postoperative complications and the high success rate of this technique provide reason to consider LLS as an option to treat apical and anterior POP.

This is the first study on LLS with a clinical follow up of two years (mean follow up of 20 months); the majority of previous studies on LLS have a 12 month-follow-up with objective evaluation [19,21], while the only study with a mean follow up of 7.2 years is based on telephone interviews on patients' satisfaction $[10,28]$.

Weakness of the present study is the presence of different minimal invasive approaches and differences in type of sutures utilized. A multicenter randomized controlled study is ongoing, comparing standardized LLS and SCP for the treatment of apical defects.

\section{Conclusion}

This series of LLS with mesh procedures for the treatment of anterior and apical prolapse performed by two institutions showed that the technique is feasible and reproducible with optimal results in term of safety and effectiveness, after 2 years follow up. However, randomized controlled studies are needed to clarify if this technique can be considered an alternative in the treatment of POP.

\section{Founding}

No founding sources supporting the work or institutional or corporate affiliations of the authors.

\section{Declaration of Competing Interest}

None.

\section{Acknowledgements}

None.

\section{References}

[1] Nygaard I, Barber MD, Burgio KL, Kenton K, Meikle S, Schaffer J, et al. Prevalence of symptomatic pelvic floor disorders in US women. JAMA 2008;300:1311-6.

[2] Barber MD, Maher C. Apical prolapse. Int Urogynecol J 2013;24:1815-33.

[3] Neuman M, Lavy Y. Conservation of the prolapsed uterus is a valid option: medium term results of a prospective comparative study with the posterior intravaginal slingoplasty operation. Int Urogynecol J Pelvic Floor Dysfunct 2007;18:889-93.

[4] Smith FJ, Holman CD, Moorin RE, Tsokos N. Lifetime risk of undergoing surgery for pelvic organ prolapse. Obstet Gynecol 2010;116:1096-100.
[5] Vieillefosse S, Thubert T, Dache A, Hermieu JF, Deffieux X. Satisfaction, quality of life and lumbar pain following laparoscopic sacrocolpopexy: suture vs. tackers. Eur J Obstet Gynecol Reprod Biol 2015;187:51-6.

[6] Higgs A, Chua HL, Smith AR. Long term review of laparoscopic sacrocolpopexy. BJOG 2005;112:1134-8.

[7] Propst K, Tunitsky-Bitton E, Schimpf MO, Ridgeway B. Pyogenic spondylodiscitis associated with sacral colpopexy and rectopexy: report of two cases and evaluation of the literature. Int Urogynecol J 2014;25:21-31.

[8] Kapandji M. Treatment of urogenital prolapse by colpo-hystmo-cydtopexy with transverse strip and crossed, multiple layer, ligamento-peritoneal douglasorraphy. Ann Chir 1967;21:321-8.

[9] Dubuisson JB, Chapron C. Laparoscopic iliac colpo-uterine suspension for treatment of genital prolapse, using two meshes. A new operative technique. J Gynecol Surg 1998;14:153-9.

[10] Veit-Rubin N, Dubuisson JB, Gayet-Ageron A, Lange S, Eperon I, Dubuisson J. Patient satisfaction after laparoscopic lateral suspension with mesh for pelvic organ prolapse outcome report of a continuous series of 417 patients. Int Urogynecol J 2017;28:1685-93.

[11] Mereu L, Terreno E, Dalprà F, Tateo S. Mini-laparoscopic lateral suspension repair of apical pelvi organ prolaps. Gynecol Surg 2017;(suppl 1):ES26-273.

[12] Simoncini T, Russo E, Mannella P, Giannini A. Robotic-assisted apical lateral suspension for advanced pelvic organ prolapse: surgical technique and perioperative outcomes. Sug Endosc 2016;30:5647-55.

[13] Cassandra L, Carberry MD, Tulikangas Paul K, Ridgeway Beri M, Collins Sarah A Rony AA. American urogynecologic society best practice statement: evaluation and counseling of patients with pelvic organ prolapse. Female Pelvic Med Reconstr Surg 2017;23:281-7.

[14] Shumaker SA, Wyman JF, Uebersax JS, McClish D, Fantl JA. Health-related quality of life measures for women with urinary incontinence: the Incontinence Impact Questionnaire and the Urogenital Distress Inventory. Continence Program in Women (CPW) Research Group. Qual Life Res 1994;3 (October 5):291-306.

[15] Agachan F, Chen T, Pfeifer J, Reissman P, Wexner SD. A constipation scoring system to simplify evaluation and management of constipated patients. Dis Colon Rectum 1996;39(June 6):681-5.

[16] Rosenthal R, Hoffmann H, Clavien PA, Bucher HC, Dell-Kuster S. Definition and classification of intraoperative complications (CLASSIC): Delphi study and pilot evaluation. Word J Surg 2015;39:1663-71.

[17] Clavien PA, Barkun J, de Oliveira ML, et al. The Clavien- Dindo classification of surgical complications: five-year experi-ence. Ann Surg 2009;250:187-96.

[18] Haylen BT, Freeman RM, Swift SE, Cosson M, Davila GW, Deprest J, et al. An International Urogynecological Association (IUGA)/International Continence Society (ICS) joint terminology and classification of the complications related directly to the insertion of prostheses (meshes, implants, tapes) and grafts in female pelvic floor surgery. Neurourol Urodyn 2011;30(January):2-12.

[19] Dubuisson JB, Yaron M, Wenger JM, Jacob S. Treatment of genital prolapse by laparoscopic lateral suspension using mesh: a series of 73 patients. J Minim Invasive Gynecol 2008;15:49-55.

[20] Dalprà F, Mereu L, Del Pezzo C, Nozza E, Perin E, Tateo S. Laparoscopic protesic lateral suspension for genital prolaps: a modified apporach. Gynecol Surg 2013;10(suppl 1):S1-S151.

[21] Dubuisson J, Eperon I, Dällenbach P, Dubuisson JB. Laparoscopic repair of vaginal vault prolaps by lateral suspension mesh. Arch Gynecol Obstet 2013;287:307-12

[22] Schug-Pass C, Tamme C, Tannapfel A, Köckerling FA. Lightweight polypropylene mesh (TiMesh) for laparoscopic intraperitoneal repair of abdominal wall hernia: comparison of bicompatibility the Dual Mesh in an experimental study using the porcine model. Surg Endosc 2006;20:402-9.

[23] Horstman R, Hellwing Classen C, Röttgermann S, Palmes D. Impact of polypropylene using pure, mixed, and titanium -coated meshes. World J Surg 2006;30:1747-9.

[24] Kockerling F, Schug-Pass C. What do we know about titanized polypropylene meshes? An evidence-based review of the literature. Hernia 2014;18:445-57.

[25] Dallenbacht P, De Oliveira SS, Marras L, Boulvain M. Incidence and risk factor for mesh erosion after laparoscopic repair of pelvic organ prolapse by lateral suspension with mesh. Int Urogynecol J 2016;27:1347-55.

[26] Davila GW, Drutz H, Deprest J. Clinical implications of the biology of grafts: conclusions of the 2005 IUGA grafts roundtable. Int Urogynecol J Pelvic Floor Dysfunct 2006;17(Suppl 1):S51-5.

[27] Le TH, Kon L, Bhatia NN, Ostergard DR. Update on the utilization of grafts in pelvic reconstruction surgeries. Curr Opin Obstet Gynecol 2007;19(5):480-9.

[28] Veit-Rubin N, Dubuisson JB, Lange S, Eperon I, Dubuisson J. Uterus-preserving laparoscopic lateral suspension with mesh for pelvic organ prolapse: a patientcentered outcome report and video of a continuous series of 245 patients. Int Urogynecol J 2016;27:491-3.

[29] Miedel A, Tegerstedt G, Morlin B, Hammarstrom M. A 5-year prospective follow-up study of vaginal surgery for pelvic organ prolaps. Int Urogynecol J Pelvic Floor Dysfunct 2008;19:1593-601.

[30] Diwadkar GB, Barber MD, Feiner B, Maher C, Jelovsek JE. Complication and reoperation rates after apical vaginal prolapse surgical repair: a systematic review. Obstet Gynecol 2009:113:267-373.

[31] Sze EH, Meranus J, Kohli N, Miklos JR, Karram MM. Vaginal configuration on MRI after abdominal sacrocolpopexy and sacrospinous ligament suspension. Int Urogynecol J Pelvic Floor Dysfunct 2001;12:375-9. 